\title{
Modell zur Übertragbarkeit der Ergebnisse aus der Feldstudie auf größere Regionen
}

\section{Berücksichtigung aktueller technischer und organisatorischer Rahmenbedingungen beim Einsatz autonomer Shuttlebusse}

\author{
Jane Wuth und Wolfgang Dorner
}

Heutzutage sind automatisierte Shuttlebusse noch eher selten im öffentlichen Straßenverkehr zu finden. Feldstudien sind daher unumgänglich, um eine Analyse der existierenden Möglichkeiten und nötigen technischen Maßnahmen zur Integration dieser Fahrzeuge in den öffentlichen Personennahverkehr (ÖPNV) zu erproben. Da es sowohl technisch als auch rechtlich noch Einschränkungen der Nutzung automatisierter Fahrzeuge gibt, stellt sich die Frage, ob potenzielle Routen, die diesen Rahmenbedingungen gerecht werden, automatisiert ermittelt werden können. Allerdings ist die Erstellung eines Modells zur Identifikation von Strecken für automatisierte Fahrzeuge unter den gegebenen rechtlichen Randbedingungen noch nicht näher betrachtet worden. Die hier verwendeten Parameter sind auf Grundlage der Ergebnisse einer Studie in Bad Birnbach, Bayern entstanden. Im Vergleichsraum Bayern wurden ca. 100 Strecken identifiziert, auf welchen man einen automatisierten Shuttlebus im derzeitigen technisch-rechtlichen Rahmen weiter testen könnte. Die sich stetig weiterentwickelnde Technologie und die teils unvollständige Attribuierung der verwendeten Daten (offizielle Verkehrsdaten ATKIS, OpenStreetMap aus dem Jahr 2018) erfordern eine Überprüfung der Ergebnisse vor Ort sowie eine laufende Fortschreibung des Modells.

\subsection{Einleitung}

Seit 2016 werden erste Erprobungen mit automatisierten Bussen im öffentlichen Personennahverkehr (ÖPNV) durchgeführt. Besonders hervorzuheben ist dabei eine Studie in Sitten, Schweiz, wo eine Strecke von 1,5 km durch die Altstadt und Fußgängerzone führt.

J. Wuth · W. Dorner $(\bowtie)$

Technische Hochschule Deggendorf, Deggendorf, Deutschland

E-Mail: jane.wuth@th-deg.de; wolfgang.dorner@th-deg.de 
Angesichts der Vielzahl an positiven Rückmeldungen soll das Projekt auf zwei weitere Schweizer Städte ausgeweitet werden (Postauto Schweiz 2017). Seit 2018 werden vermehrt andernorts automatisierte Shuttlebusse getestet. Dazu gehören auch Strecken in Metropolregionen wie Helsinki oder Paris (Mogg 2017). Der automatisierte Bus in Bad Birnbach war der erste seiner Art in Deutschland. Gerade die lange Dauer des Testbetriebs machen die erhobenen Daten einzigartig. Aufgrund der Erfahrungen, welche in den vorhergehenden Kapiteln detailliert beschrieben wurden, werden hier nun die Übertragbarkeit der Erkenntnisse aus der Studie in Bad Birnbach auf andere Regionen Bayerns und die Kriterien zur automatisierten Auswahl solcher Strecken analysiert. Mit ihren Herausforderungen, wie Straßenunterführungen oder wechselnde Umgebungen, ermöglicht es die Route in Bad Birnbach, die gesammelten Informationen anzuwenden, um ähnliche Strecken zu identifizieren und gleichzeitig solche mit neuen Arten von Hindernissen zu finden. Somit können Erfahrungen aus Bad Birnbach übertragen und gleichzeitig die Weiterentwicklung der Verfahren forciert werden. Das Ziel der Streckenfindung liegt darin, eine für den Bus befahrbare Route zu finden, aber gleichzeitig ggf. den Schwierigkeitsgrad zu steigern. Nur so kann die noch relativ neue Technologie weiterentwickelt und an die Straßenverhältnisse angepasst werden.

Trotz der wachsenden Zahl an Teststrecken, auf denen automatisierte Busse getestet werden, stecken die rechtlichen und technischen Rahmenbedingungen noch in ihren Kinderschuhen. Das Wiener Übereinkommen im Straßenverkehr von 1968 ist noch heute Basis der allgemeinen Verkehrsgesetze und durch die damals herrschenden Umstände noch nicht an neue Technologien angepasst. Daher ist die eigentliche Straßenzulassung automatisierter Fahrzeuge beschränkt und Versuche im öffentlichen Nahverkehr somit stark eingeschränkt. Viele technische Möglichkeiten des Busses können deswegen nicht erprobt werden.

Dieser Beitrag ist besonders auf die Fragestellung fokussiert, wie technische, rechtliche und auch organisatorische Bedingungen den Einsatz automatisierter Shuttlebusse räumlich einschränken können. Dazu entsteht die Frage, wie Ergebnisse aus einer Modellregion wie Bad Birnbach übertragen werden können und wo ein Einsatz automatisierter Shuttlebusse in Bayern noch denkbar wäre. Denkt man über die Routenplanung und Einsetzbarkeit von Shuttlebussen sowie ihre organisatorischen Einschränkungen nach, ergibt sich die Frage, wie man solche Fahrzeuge alternativ einsetzen könnte und wie sich dieser Einsatz unter sich verändernden Rahmenbedingungen weiterentwickeln lässt.

Ziel des Vorhabens ist damit, die Ergebnisse der vorangegangenen Kapitel aufzugreifen und die Übertragbarkeit des Konzepts eines automatisierten Shuttlebusses sowie dessen Einsatz in neuen Regionen zu analysieren.

Der Beitrag gliedert sich wie folgt: Das nächste Kapitel fasst die derzeitige Lage der Pilotprojekte mit automatisierten Shuttles, deren Einschränkungen und Potenziale, sowie die allgemeine Literatur um automatisierte Fahrzeuge zusammen. Im darauffolgenden Kapitel werden der Modellansatz und das verwendete Material vorgestellt, um anschließend die Ergebnisse anhand von Karten zu präsentieren. Abschließend werden die Ergebnisse und Möglichkeiten der Übertragbarkeit diskutiert sowie ein Ausblick auf eine Fortschreibung des Ansatzes gegeben. 


\subsection{Literatur}

In Sitten in der Schweiz fährt seit 2016 ein automatisiertes Shuttle im öffentlichen Personennahverkehr. Dieser nutzt allerdings keine öffentlichen Straßen, sondern bedient die Fußgängerzonen und Altstadtgebiete. Die Strecke hat eine Gesamtlänge von 1,5 km, welche nach dem ersten Jahr in den städtischen Personennahverkehr vollwertig miteinbezogen werden sollte (Postauto Schweiz 2017). In Deutschland ist der Shuttlebus in Bad Birnbach der erste seiner Art mit einer gültigen Straßenzulassung. Ein vergleichbares Projekt ist beispielsweise TaBuLa in Hamburg, ein Testzentrum für automatisierte Busse im Kreis Herzogtum Lauenburg (TaBuLa 2018). Hier sollen erstmals automatisierte Busse (ebenfalls mit Operator, wie in Bad Birnbach) in der zweiten Jahreshälfte 2019 mit Fahrgästen fahren. In Wien, Österreich wurde das Kooperationsprojekt „,auto.Bus-Seestadt“ initiiert. (L o. J.). Der automatisierte Kleinbus wird seit 6. Juni 2019 auf öffentlichen StraBen im Personennahverkehr eingesetzt (Wiener Linien 2019). Im Land Kärnten, ebenfalls in Österreich, wurde im Juni 2018, erstmals ein automatisierter Bus für eine kurze Pilotphase auf den öffentlichen Straßen genutzt und auch in Helsinki und Paris (Mogg 2017) sind seit 2017 automatisierte Busse zu Versuchszwecken im Einsatz.

Projekte, die keine Straßenzulassung erhalten, weichen oft auf private Gelände wie beispielsweise den Campus Charité Mitte in Berlin (Neumann 2017) aus. Durch die beschränkten Zulassungsmöglichkeiten für automatisierte Fahrzeuge zum Schutz der regulären Verkehrsteilnehmer sind private größere Gelände gute Ausweichstrecken, um weiter an der Technologie arbeiten zu können.

Die steigende Zahl an Feldstudien zu automatisierten Shuttlebussen zeigt, dass derzeit die Literatur und Datensammlung zu diesem Thema noch sehr limitiert ist. Andererseits ist es ein Zeichen dafür, dass diese noch sehr neue Technologie bald marktreif wird und es nicht mehr lange dauern wird, bis der automatisierte Shuttlebus gleichberechtigt zu anderen Technologien im öffentlichen Personennahverkehr agieren kann. Was allerdings noch an die neuen Veränderungen angepasst werden muss, sind betroffene Normen und rechtliche Randbedingungen für automatisierte Fahrzeuge.

Mitte des Jahres 2018 hat die deutsche Bundesregierung einen Bericht zum rechtlichen Rahmen des automatisierten Fahrens auf deutschen Verkehrsstraßen veröffentlicht (wiss. Dienste Deutscher Bundestag 2018). In diesem wird die Rechtslage auf die fünf Stufen des autonomen Fahrens angewendet. Im Fall eines automatisierten Shuttles ist das Ziel, dass das Fahrzeug komplett ohne Fahrzeugführer auskommen kann, somit Stufe 5, die Vollautomatisierung (siehe Kap. 5), erreicht wird. Die derzeitige rechtliche Lage sieht allerdings vor, dass in jedem Fall ein Fahrzeugführer im Shuttle anwesend ist, der jederzeit eingreifen können muss, sollte das Fahrzeug Situationen falsch einschätzen. Zusätzlich sind autonome Lenkanlagen noch vollständig verboten, wenn das Fahrzeug eine Geschwindigkeit von über $12 \mathrm{~km} / \mathrm{h}$ erreicht. Dies ist allerdings notwendig, wenn man einen automatisierten Shuttlebus ohne Fahrzeugführer einführen möchte. Das Wiener Übereinkommen zwischen den Mitgliedsstaaten der Vereinten Nationen (VN) aus dem Jahr 1968, das noch heute Grundlage aller Verkehrsgesetze in Deutschland ist, wurde Mitte des 
Jahres 2016 modifiziert. Nachdem das Straßenverkehrsgesetz in Deutschland 2017 nun auch für automatisiertes Fahren angepasst wurde, ist der Grundstein für die rechtliche Zulassung automatisierter Shuttlebusse gelegt. Es wurde festgelegt, dass in Fahrzeugen Automatisierungstechniken verwendet werden dürfen, solange diese vom Fahrzeugführer jederzeit übersteuert werden können.

\subsection{Modelle für Routenberechnung und Planung im ÖPNV}

Das Thema Routenplanung selbst kann von unterschiedlichsten Seiten beleuchtet werden. Eine der Grundlagen im vorliegenden Fall ist das Vehicle-Routing-Problem (VRP), um die effiziente Nutzung eines oder mehrerer Fahrzeuge zur Abholung von Gütern oder Personen an mehreren Stationen zu berechnen. Die Literatur und die Anzahl an Unterarten des VRPs ist immens, dreht sich allerdings vorwiegend um den Transport von Gütern und weniger um Personentransporte (Koç et al. 2016; Braekers et al. 2016).

Die Organisation des öffentlichen Personennahverkehrs unterliegt verschiedenen Organisationen auf unterschiedlichen räumlichen Gliederungsebenen. Daher hat jedes Verkehrsnetz ein eigenes System als Grundlage, es wird auf die örtlichen Gegebenheiten geachtet und das Liniennetz größtenteils manuell erstellt. Strukturierungen des ÖPNV durch Modelle, welche regionsübergreifend anwendbar sind, sind dementsprechend rar (Meignan et al. 2007). In jeder Stadt oder Region gibt es verschiedene Herausforderungen, die individuelle Lösungen benötigen. Überregionale Modelle sind aufgrund der auftretenden Komplexität, selten zu finden.

Möchte man das Routing für den ÖPNV mit automatisierten Fahrzeugen modellieren, kommt hinzu, dass ohne den Fahrzeugführer geplant werden muss. Die Grundvoraussetzung für vollständig automatisiertes Fahren (also ohne jegliche Interaktion des Fahrzeugführers) ist nämlich, dass die Fahrzeuge Routeninformationen erhalten und verarbeiten können, ohne dass dies durch manuelle Tätigkeiten am Fahrzeug einzustellen ist. Deswegen beschäftigt sich ein großer Bereich um automatisierte Fahrzeuge mit dem Thema Routenplanung.

Meistens geht es allerdings darum, den Individualverkehr zu optimieren, und zu modellieren, wie einzelne Strecken zwischen zwei Punkten angefahren werden können. Zu den Ansätzen der Optimierung des Individualverkehrs zählen beispielsweise auch der Austausch von Daten zwischen automatisierten Fahrzeugen und somit das Wegfallen von Ampeln und anderen Wartezeiten (Chu et al. 2017) oder die Reservierung von ganzen Fahrbahnen rein für automatisierte Fahrzeuge (Talebpour et al. 2017).

Spichkova et al. (2015) präsentieren ein Modell, mit welchem intelligente Routen im ÖPNV generiert werden können. Dieses Modell beschreibt, wie für das automatisierte Fahrzeug zwischen verschiedenen Haltestellen eine Route erstellt wird. Damit kann das Fahrzeug ohne menschliche Interaktion die zu befahrene Strecke je nach Anfragen der Mitfahrer planen. Hierbei wird im Modell vorgesehen, dass fixe Haltestellen existieren und auch nur diese angefahren werden können, allerdings nur nach Buchung durch einen 
potenziellen Fahrgast. Überflüssige Halte werden dadurch vermieden. Nachteilig an diesem Modell ist die sehr zukunftsorientierte technologische und rechtliche Ausrichtung. Heutige Randbedingungen, wie Restriktionen bei der Streckenwahl, finden bei Spichkova et al. (2015) keine Berücksichtigung.

Bleibt man in der zukünftigen Planung des öffentlichen Personennahverkehrs, gibt es unterschiedliche Strukturmodelle. Csiszár und Zarkeshev (2017) unterscheiden in der Planung von Bushaltestellen und Routen zwischen zwei Ansätzen. Der erste Ansatz erklärt, dass man die derzeitige Straßeninfrastruktur beibehalten sollte und Busse durch automatisierte Fahrzeuge leicht ersetzen kann. Dabei wird in den Vordergrund gestellt, dass nur konventionelle Fahrzeuge Unfälle mit automatisierten Bussen verursachen könnten. Deswegen wäre anzustreben, den kompletten Straßenverkehr durch automatisierte Fahrzeuge zu ersetzen und die Infrastruktur wie heute bestehen zu lassen.

Der zweite Ansatz nach Csiszár and Zarkeshev (2017) basiert auf der Methode des Automated Demand-Responsive Transportation Service (ADRTS), welcher individuelle Routen und Haltepunkte je nach Anfrage anfährt. Hierbei gibt es somit zwar teilweise fixe Haltestellen, allerdings sind diese nur bei Bedarf anzufahren. Notwendig für ein funktionierendes Transportsystem dieser Art ist die Kommunikation zwischen automatisiertem Bus und den anderen der Flotte angehörigen Fahrzeugen, sowie dem Fahrgast.

Geht es an die Überlegungen der optimalen Nutzung automatisierter Fahrzeuge im öffentlichen Verkehr, wird vermehrt auf die Entwicklung automatisierter Car-SharingModelle gesetzt, also der Reduktion des Besitzes von privaten Pkws und der Expansion des Car-Sharings durch automatisierte Fahrzeuge (Cyganski 2015; Lenz and Fraedrich 2015; Heilig et al. 2017). Heilig et al. (2017) haben hierfür ein Modell unter eher radikalen Annahmen entwickelt. Sie gehen von einer Welt ganz ohne private Fahrzeuge aus, um dann die Nachfrage von und Abdeckung durch autonomous mobility on demand (AMOB), also autonome Fahrzeuge auf Nachfrage, zu errechnen. Ihre Resultate zeigen, dass der AMOB-Service zwar den Großteil der Nachfrage abdecken kann, die Nutzung von Fahrrad- oder Fußwegen und öffentlichem Nahverkehr steigt aber gleichzeitig. Diese Theorie basiert auf sehr weitreichenden Annahmen, wie der Inexistenz privater Autos und einer entsprechend entwickelten Gesetzeslage und technologischem Fortschritt. Dia and Javanshour (2017) gehen in ihrer Modellierung ebenfalls von einem AMOB-Service aus, allerdings sind sie etwas näher an der derzeitigen Realität, da sie drei verschiedene Szenarien vergleichen; eines ohne autonome Fahrzeuge, und zwei mit zusätzlichen privaten und öffentlichen autonomen Shuttles sowie unterschiedlichen Wartezeiten. In ihren Ergebnissen verdeutlichen sie, dass die zwei Szenarien mit autonomen Fahrzeugen weniger Verkehr produzieren und weniger Parkplätze benötigen. Im Vergleich dazu gehen Lenz and Fraedrich (2015) davon aus, dass durch autonomes Fahren, die Möglichkeiten des Car-Sharings zum einen vielfältiger werden und zum anderen die Angebote des heutigen ÖPNV vervollständigen. Es könnte beispielsweise das Fahrzeug entweder als Vehicle-On-Demand genutzt werden oder um den öffentlichen Verkehr zu flexibilisieren. Hierbei sind die einzelnen Theorien sehr ähnlich zueinander und unterscheiden sich meistens nur in Details. 
Bisher veröffentlichte Studien weisen eine relativ große Lücke zwischen dem heutigen Einsatz von herkömmlichen öffentlichen Fahrzeugen des Personennahverkehrs und dem zukünftigen Einsatz von autonomen Shuttles auf. Wenig erforscht wurde, wie unter den heutigen Umständen autonome Fahrzeuge im ÖPNV genutzt werden können. Bis dato wird in der Regel von einem voll autonomen Fahrzeug ausgegangen, sowie einem gesetzlich wenig regulierten Markt- und Verkehrssystem. In der Realität sind autonome Fahrzeuge allerdings sowohl technisch als auch rechtlich in ihren Einsatzmöglichkeiten noch sehr beschränkt. Es stellt sich damit die Frage, wo bereits heute ein Einsatz möglich ist und die aktuellen rechtlichen und technischen Restriktionen nicht relevant sind bzw. heutige Standards eingehalten werden können.

\subsection{Material und Methode}

Die verhältnismäßig lange Dauer der Studie in Bad Birnbach ermöglichte es, neben technischen Faktoren des Einsatzes eines automatisierten Busses auch die reale Erfahrung von Fahrgästen zu untersuchen (siehe Kap. 6 und 9). Dies bietet besondere Möglichkeiten, aus den Ergebnissen der Studie die relevanten Faktoren und Parameter für eine Übertragbarkeit auf andere Regionen zu identifizieren und weiterzuverarbeiten. Dadurch kann der Einsatz des automatisierten Busses auf heutige Möglichkeiten und Bedürfnisse angepasst werden. Die Übertragbarkeit der Ergebnisse bezieht sich zum einen auf die (zugelassenen) Fähigkeiten und die technischen Eigenschaften des Shuttles und zum anderen darauf, wie man den Shuttlebus in Zukunft noch besser an die derzeit herrschenden technischen Randbedingungen und artikulierten Bedarfe anpassen kann. Das Übertragen der Ergebnisse aus der Feldstudie hat also zum Ziel, mögliche weitere Routen für automatisierte Fahrzeuge zu identifizieren.

Ganz nach der requirements-error-taxonomy-Theorie von Anu et al. (2016) soll eine neue Route für das automatisierte Shuttle zwar befahrbar sein, aber gleichzeitig auch gezielt neue Herausforderungen beinhalten, an denen die Technik überprüft und ggf. verbessert werden kann.

Die hier angestrebte Analyse ist auf den Vergleichsraum Bayern beschränkt. Bayern besteht aus unterschiedlich strukturierten Städten und weitflächigen ländlichen Räumen, wodurch sehr verschiedene Straßenverhältnisse, Siedlungsgrößen und Siedlungsstrukturen sowie Topographien existieren. Dadurch kann das Routing-Verfahren zur Identifikation unterschiedlicher Strecken unter Berücksichtigung örtlicher Gegebenheiten entwickelt und getestet werden. Gleichzeitig wird durch die Begrenzung auf Bayern eine zwar große, aber noch zu verarbeitende Menge an Daten genutzt.

Die Eingangsparameter sowie Grundlagen des Modells wurden zuerst auf Basis von drei Experteninterviews sowie Berichten der anderen Teilprojekte (Kap. 3-9) gestützt. In einem zweiten Schritt werden diese Randbedingungen auf räumliche Parameter übertragen. Einige der beeinflussenden Faktoren sind dennoch nur teilweise anwendbar und auf Grundlage räumlicher Analysen bearbeitbar, da Informationen, wie beispielsweise die Verkehrsdichte von Radfahrern auf der Straße, nicht flächendeckend dokumentiert sind. 
Die in der Studie identifizierten relevanten Parameter sind:

Straßenbreite: Das Fahrzeug selbst hat eine Breite von knapp 2 m. Dazu kommt der Sicherheitsradius, der auf allen Seiten des Shuttles jeweils $150 \mathrm{~cm}$ beträgt. Einschränkungen dieses Lichtraumprofils verursachen eine Geschwindigkeitsreduktion bzw. Gegenstände innerhalb eines Sicherheitsradius von $30 \mathrm{~cm}$ führen zu einem Stopp des Shuttles. Liegt die Fahrbahnbreite also unter 5 m, kann es dazu kommen, dass beispielsweise entgegenkommende Fahrzeuge in den Sicherheitsbereich des Shuttlebusses geraten und so zur Reduktion der Fahrgeschwindigkeit führen.

Deckenhöhe bei Tunneln: Mit seinen 2,75 m Höhe und dem 150 cm großen Sicherheitsradius kann das Shuttle nur Tunnels oder andere Unterführungen durchfahren, wenn diese eine minimale Deckenhöhe von 4,3 m haben.

Straßensteigung: Der Shuttlebus hat eine maximale Geschwindigkeit von $40 \mathrm{~km} / \mathrm{h}$ und eine Fahrgeschwindigkeit von $20 \mathrm{~km} / \mathrm{h}$. Aufgrund dieser eingeschränkten Leistungswerte sind gewisse Straßensteigungen für ihn noch nicht zu bewältigen. In den Interviews und als Grundlage für die Planung der Strecke in Bad Birnbach wurde eine Steigung von nicht mehr als $5 \%$ gewählt.

Distanz: Durch die relativ langsame Geschwindigkeit des Fahrzeugs ist es noch unrentabel, eine Strecke von über 5 km Länge anzubieten, da die Fahrgäste von Start bis Ziel länger als eine Stunde im Bus mitfahren würden. Gleichzeitig ist die Batterie des Shuttles aufzuladen, was zu Unmut bei den Fahrgästen führen kann, sollte der Bus auf der Strecke zwischenladen müssen. Folglich ist im Modell der maximale Aktionsradius, ausgehend vom Startpunkt, bei der Wahl potenzieller Strecken, auf $3 \mathrm{~km}$ festgelegt und die Streckenlänge auf 4,5-5,5 km beschränkt. Die Streckenlänge sowie der Radius um den Startpunkt sind im Modell als frei wählbare Parameter definiert worden, werden aber für diese Studie auf die genannten Entfernungen eingestellt.

Beeinflussende Umgebungsfaktoren: Weitere Faktoren, die die Straßenauswahl für die Streckenberechnungen minimieren, sind die Menge an Fußgängern und Fahrradfahrern auf den es für die Positionsbestimmung des Shuttles von großer Wichtigkeit, genügend Referenzpunkte zu haben. Wie in Kap. 5 beschrieben, mussten in Bad Birnbach an einem Teil der Strecke, welcher durch eine Wiese führte, eine Beschilderung mit Extra-Referenzpunkten angebracht werden, damit der Bus seine Ortsbestimmung durchführen konnte. Es wird vermutet, dass spiegelnde Flächen (wie beispielsweise von der Sonne beschienene Schaufenster) die Ortsbestimmung für das Fahrzeug erschweren oder die Sensoren beeinträchtigt. Außerdem sind Nebel, Wasserdampf (Therme), Regen, Flimmern der Luft, etc. Faktoren, die die Sensoren hindern, die Umgebung klar zu identifizieren, was erneut zum Stillstand des Shuttles führen kann.

Start-Zielpunkte: Diese Parameter sind zentrale Elemente der Streckenbestimmung. Mögliche Start- und Zielpunkte hängen dabei eng mit potenziellen Nutzergruppen zusammen. Die geringe Geschwindigkeit des Busses lässt erwarten, dass besonders Menschen, die selbst auf kurzen Gehstrecken Schwierigkeiten haben, eine relevante Nutzergruppe darstellen. Startund Zielorte hängen damit stark mit dem aktuellen und gewünschten Mobilitätsverhalten dieser Nutzer zusammen. Die gewählten Orte werden im nächsten Kapitel detailliert erläutert. 
Die verwendeten Geobasisdaten sind ATKIS-Daten (Amtliche TopographischKartographische Informationssystem-Daten) der Bayerischen Landesvermessungsverwaltung und OpenStreetMap (OSM) Daten. OSM Daten gehören der Gruppe der Volunteered Geographic Information (VGI) an und sind kostenfreie Datensätze, welche von Nutzern der Plattform auf freiwilliger Basis erhoben und editiert werden (OpenStreetMap o. J.). Da kein groß angelegter Verifizierungsprozess hinter der Datenbeschaffung liegt, sind OSM Daten teilweise unvollständig, doppelt erfasst oder falsch klassifiziert (Valdes et al. 2019). Da diese Daten jedoch für die Start- und Endpunkte der Strecke sowie auf dem Weg liegende Points of Interest (POIs) genutzt werden, ist die Dopplung der Daten eher zu vernachlässigen. Die Klassifizierungsfehler bzw. das Fehlen gewisser POIs sind bekannte OSM-Probleme, welche bei geeigneter Berücksichtigung im Modell und durch die Breite der erfassten Information kompensiert werden.

Die verwendeten ATKIS-Daten hingegen sind aus dem amtlichen Digitalen BasisLandschaftsmodell entnommen und von der Bayerischen Vermessungsverwaltung zur Verfügung gestellt (Landesamt für Digitalisierung, Breitband und Vermessung o. J.). In diesem Datensatz sollten keine Fehler oder fehlenden Straßenabschnitte vorkommen.

Das Modell selbst wird im Geoinformationssystem ArcGIS Pro abgebildet, und auch die Berechnungen werden dort durchgeführt. Es basiert auf den beschriebenen geographisch referenzierten Daten, mit denen Netzwerke modelliert und Routen geplant werden können. Hierfür müssen die genutzten Datensätze gefiltert, auf den zu betrachtenden Bereich reduziert und dann miteinander verbunden werden.

\subsubsection{Auswahl des Straßennetzes}

Um ein geeignetes Modell zu erstellen, sind die Inputdaten von großer Bedeutung. Zur Streckenanalyse wurden ATKIS-Verkehrsdaten herangezogen (Landesamt für Digitalisierung, Breitband und Vermessung o. J.). Da das Fahrzeug auf öffentlichen Straßen fahren soll, sind private Straßen und Straßen mit Zufahrtsbeschränkungen, wie beispielsweise vom Typ Bahnverkehr oder Flugverkehrsanlage, ausgeklammert worden. Nach Analysen der einzelnen Objektarten wurden die zugehörigen Polylinien-Datensätze Straßenachse und Fahrbahnachse zur Streckenfindung herangezogen.

Die ausgewählten Objektarten wurden unter Berücksichtigung der oben definierten Parameter weiter eingegrenzt. Tab. 10.1 präsentiert einen Überblick der gewählten Daten.

Nach einer Analyse des Datensatzes BauwerkImVerkehrsbereich ist ersichtlich, dass die dort beinhalteten Informationen als Barrieren für die Streckenberechnung zu nutzen sind. Dort enthalten sind zum Beispiel Tunnel, Stege oder unterschiedliche Brückentypen, welche teilweise zu niedrig, zu schmal oder für Fahrzeuge allgemein unpassierbar sind. Daher sind alle Streckenabschnitte, die für den automatisierten Bus befahrbar wären, aus dem Datensatz eliminiert und die für den automatisierten Bus unbefahrbaren Bauwerke, wie beispielsweise Fahrradbrücken, beibehalten worden. In der Modellierung wird der bereinigte Datensatz, der nur noch unpassierbare Stellen für das Shuttle beinhaltet eingesetzt, um aufgrund dessen solche Strecken zu umgehen. 
Tab. 10.1 Auswahl der Straßendaten (eigene Darstellung)

\begin{tabular}{l|l|l|l}
\hline Objektart & Eliminierte Objekte & Gelöschte Elemente & Erhalten \\
\hline Straßenachse & $\begin{array}{l}\text { - Fernverkehr } \\
\text { - Fahrstreifenanzahl > 4 } \\
\text { - Bezeichnung: Autobahn, } \\
\text { Bundesstraße, Staatsstraße }\end{array}$ & 843.695 & 681.938 \\
\hline Fahrbahnachse & $\begin{array}{l}\text { - Fahrstreifenanzahl > 4 } \\
\text { - Bezeichnung: Autobahn, } \\
\text { Bundesstraße, Staatsstraße }\end{array}$ & 7406 & 13.744 \\
\hline BauwerkImVerkehrsbereich & $\begin{array}{l}\text { Behaltene Objekte: } \\
\text { - Steg }\end{array}$ & 47.865 & 7641 \\
\hline
\end{tabular}

Die vorliegenden Datensätze beinhalten nicht alle geometrischen Merkmale, die notwendig sind, um aufgrund der o. g. Parameter eine vollständige Analyse durchzuführen. Park- und Halteflächen im Verkehrsraum sowie Verkehrsdichten werden in keinem der vorliegenden Datensätze ausgewiesen und können daher im vorliegenden Modell nicht berücksichtigt werden. Als Konsequenz befinden sich im Datensatz einige Straßenabschnitte, welche für den automatisierten Bus voraussichtlich nicht befahrbar sind.

\subsubsection{Auswahl der Points of Interest}

Die Auswahl der relevanten Start- und Zielorte wurde mithilfe von OSM-Daten durchgeführt.

Um eine Auslastung des automatisierten Busses zu erreichen, müssen die richtigen potenziellen Fahrgäste angesprochen und höher frequentierte Orte als Start- oder Zielpunkte ausgewählt werden.

Als Grundlage für die Auswahl der Start- und Endpunkte der Route werden wegen der maximalen Fahrgeschwindigkeit von $20 \mathrm{~km} / \mathrm{h}$ sowie auf Grundlage der Ergebnisse beschrieben in Kapitel (6) eher ältere Menschen als Hauptnutzer identifiziert. Gleichzeitig werden auch Touristen als potenzielle Fahrgäste einbezogen, da der automatisierte Bus auf der einen Seite einen positiven Einfluss auf das Image der Stadt hat, und auf der anderen Seite Touristen meist mehr Zeit für eine solche „Attraktion“, wie es der automatisierte Bus durch seine Seltenheit derzeit noch ist, haben.

Dementsprechend wurden zentrale Omnibusbahnhöfe oder Hauptbahnhöfe als Startpunkte ausgewählt. Diese eignen sich insbesondere dafür, da sie am ehesten für Menschen zu erreichen sind, die außerhalb des Stadtgebietes wohnen, und dort gleichzeitig eine Ladeinfrastruktur für den Bus leicht mit eingebaut werden kann. Gleichzeitig ist der Effekt auf die Transportabdeckung, besonders für Menschen mit leichter Gehbehinderung, an einem sowieso schon vorhandenen Verkehrsknotenpunkt sehr hoch.

Als Endpunkte der Route wurden, der gleichen Logik folgend, öffentliche Orte, wie z. B. Krankenhäuser, Schlösser, Botschaften und Zoos ausgewählt. Zusätzlich könnte man Altenheime oder Einkaufszentren in weitere Analysen einbeziehen. Allerdings werden, wie oben beschrieben, OSM-Daten für die Points of Interest bzw. Transportpunkte genutzt. 
In diesen gibt es keine Kategorie, welche selektiv Altenheime in Betracht zieht, und die Kategorie „Mall“, welche Einkaufszentren beschreibt, ist nicht verlässlich.

Weiterhin wurden aus den ATKIS-Objektarten „Fahrbahnachse“ und „Straßenachse“ die gekennzeichneten Fußgängerzonen extrahiert und als separate Points of Interest angelegt.

\subsubsection{Modellierung}

Um das Modell berechnen zu können, mussten, wie oben beschrieben, zuerst die relevanten Daten aus den vorhandenen Datensätzen extrahiert werden. Dazu wurde mithilfe des GIS-Programms ArcGIS Pro, durch die vorgegebene Attribuierung der Daten, der Teil des Datensatzes isoliert, welcher für die Routenberechnung nach obigen Kriterien relevant ist.

Es wurde vor der Erstellung des Routenmodells festgelegt, dass sich die vom automatisierten Bus zu befahrene Strecke in einem Radius von $3 \mathrm{~km}$ zum Startpunkt bewegen soll. Dementsprechend wurde um jeden selektierten Startpunkt ein Puffer von $3 \mathrm{~km}$ gelegt und nur die Daten für die Analyse selektiert, welche sich in diesem Radius befanden. Alle Daten, die außerhalb dieses Radius lagen, wurden eliminiert. Bei Überschneidungen mehrerer Radien mussten resultierende fehlerhafte Extraktionen nachträglich selektiert werden. Abschließend wurde der ATKIS-Vektordatensatz Verkehr in einen routingfähigen Datensatz als Netz konvertiert.

Die Netzwerkfähigkeit des Datensatzes befähigt das Programm, mögliche Routen zwischen unterschiedlichen Start- und Endpunkten zu analysieren und Routenparameter zu berechnen. Danach konnten auf unterschiedliche Arten Routen erstellt und ausgewählt werden. Da die Länge der Route im Routing-Algorithmus nicht beeinflussbar ist, wurde nachträglich eine Streckenselektion vorgenommen. Hierzu wurden nur diejenigen Strecken ausgewählt, die eine Gesamtstreckenlänge von 4,5-5,5 km haben.

\subsection{Ergebnisse}

In ganz Bayern wurden insgesamt 124 Strecken ermittelt, welche auf Grundlage der hier genutzten Daten für den automatisierten Bus eine mögliche Route darstellen könnten. Abb. 10.1 spiegelt die verwendete Datengrundlage wider. Bei der Datenselektion sind 1270 Bahnhöfe als mögliche Startpunkte ausgewählt, 1129 Zielorte der Kategorien Schloss, Krankenhaus, Botschaft und Zoo selektiert und 695.682 Straßenabschnitte für das Netzwerk verwendet worden. Zusätzlich wurden 7641 Barrieren in Form von Bauwerken im Verkehrsbereich in die Modellierung mit aufgenommen. Insgesamt wurden initial 2109 Strecken durch das Modell identifiziert. Durch die Reduktion der passenden Strecken auf die Routenlänge von 4,5-5,5 km ergibt dies eine finale Anzahl von 124 Routen.

Wie in Abb. 10.1 erkennbar, ist um die einzelnen Bahnhöfe der 3 km Radius gelegt, in welchem sich die gewählte Route befinden soll. Durch die Größe des insgesamt betrachteten 


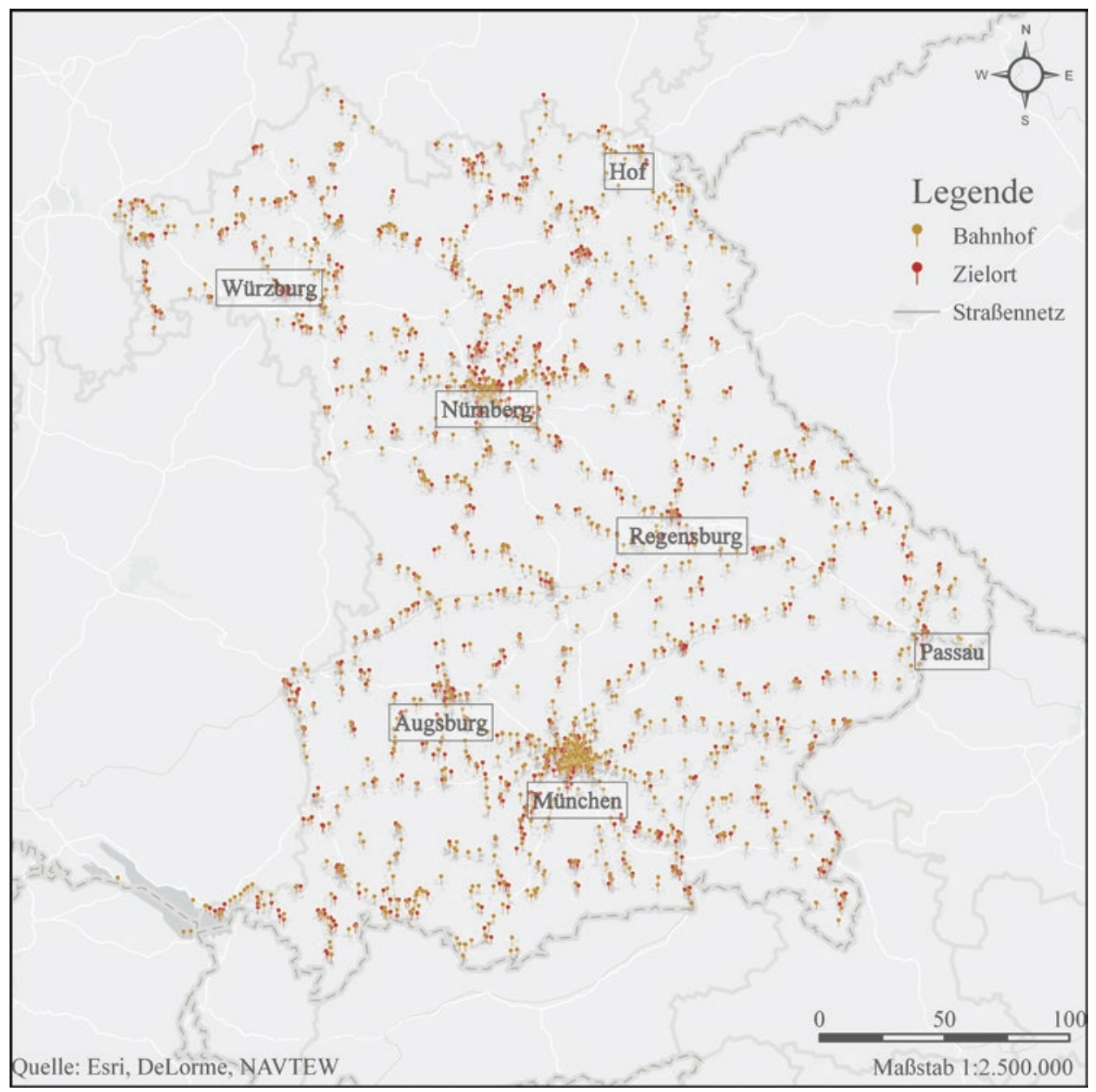

Abb. 10.1 Ausgewählte Straßen und Zielorte im Radius von $3 \mathrm{~km}$ um die Bahnhöfe als Startorte in Bayern

Gebiets sind die genauen Radien und ihre unterschiedlichen Strukturen schwer erkennbar. Vergleicht man die Metropolregionen wie München oder Nürnberg mit den eher ländlichen Regionen zwischen diesen Städten, erkennt man die Herausforderung der Überschneidung von Radien unterschiedlicher Startorte. Je besiedelter das betrachtete Gebiet ist, desto mehr Bahnhöfe gibt es.

Dies ist leicht am Beispiel von Abb. 10.2 zu erkennen. Die $5 \mathrm{~km}$ lange Route vom Bahnhof Gersthofen bis zum Krankenhaus Josefinum im Norden von Augsburg wird dort dargestellt.

Bei einer Gesamtlänge von 5 km verläuft die Strecke sehr gerade und fast ohne Abbiegen oder Kurven vom Bahnhof zum Krankenhaus. Der vorgegebene Aktionsradius des Busses von $3 \mathrm{~km}$ wird deutlich überschritten, da direkt an der Strecke ein weiterer Bahn- 
Abb. 10.2 Route vom Bahnhof Gersthofen

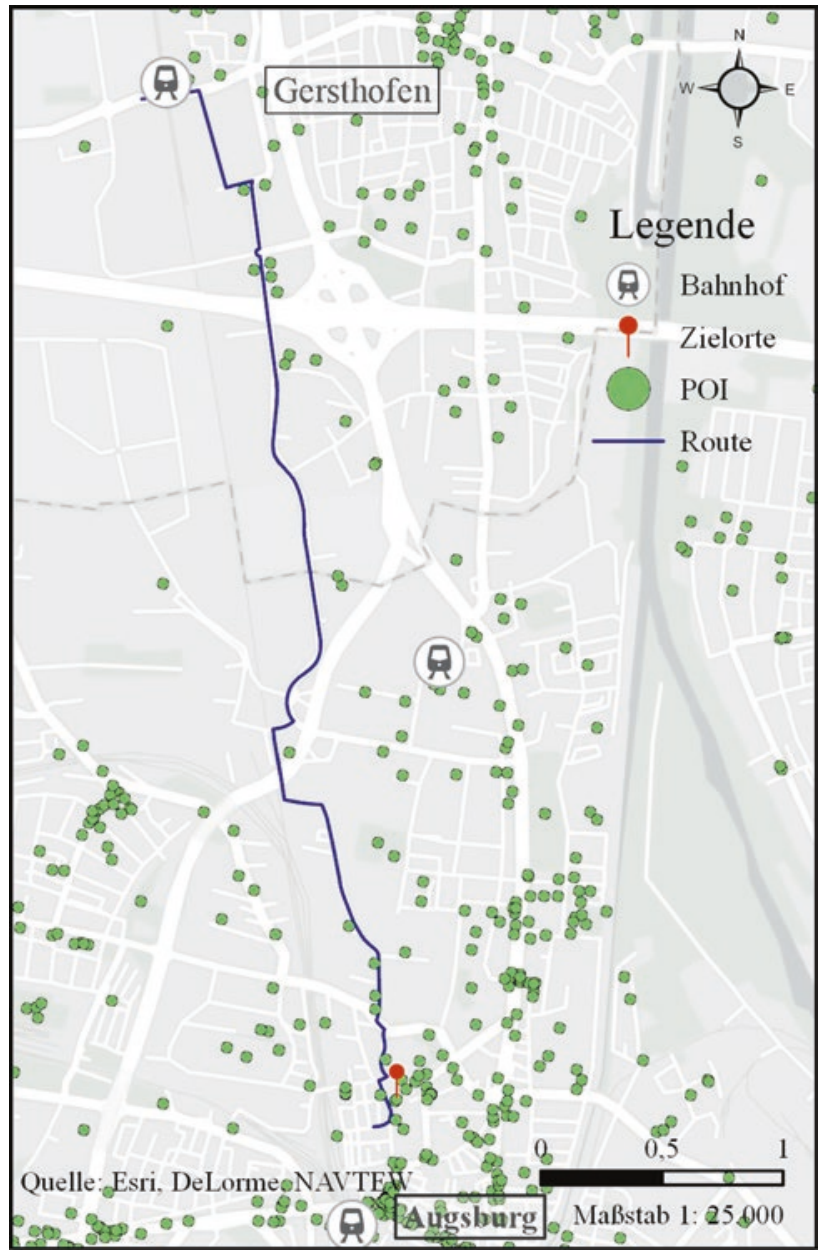

hof gelegen ist. Damit ist das verfügbare Streckennetz im Modell eigentlich das Netz von zwei sich überschneidenden Radien als Einzugsgebiete zweier Bahnhöfe.

Durch eine nachfolgende Auswahl der Routen auf Strecken mit einer Gesamtlänge von unter 5,5 km konnten solche Situationen, die ggf. zu fehlerhaften, weil zu langen Routen führen, erkannt und berücksichtigt werden.

Vergleicht man Abb. 10.2 mit der Beispielroute in Abb. 10.3, erkennt man viele Unterschiede. Auch hier überlagerten sich die Radien Bischofswiesen und Bahnhof Berchtesgaden. Allerdings ist die Route hier kurviger. Außerdem werden vom Bahnhof Bischofswiesen gleich zwei Strecken mit verschiedenen Endhaltestellen vorgeschlagen. Aus diesen zwei Routen könnte man dementsprechend eine im Kreis führende Fahrstrecke erstellen, indem man beide Ziele miteinander verbindet. Allerdings führt ein Teil der Route über eine Gemeindeverbindungsstraße, welche mit einer Geschwindigkeit von bis zu 100 km/h befahren werden darf. Der automatisierte Bus mit seiner eher geringen Fahrgeschwindigkeit 


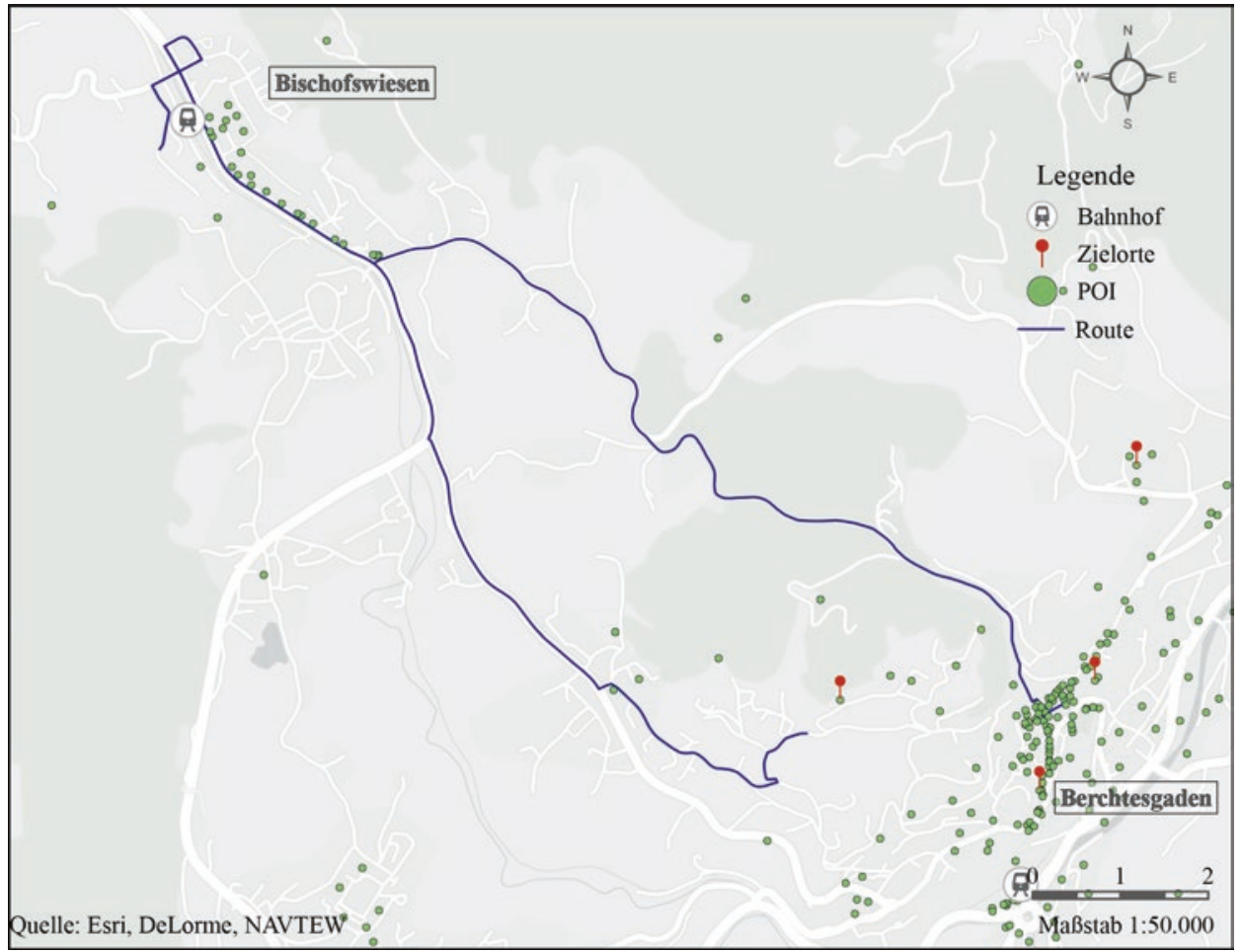

Abb. 10.3 Zwei mögliche Routen von Bischofswiesen nach Berchtesgaden

ist somit dort eher schwierig einzusetzen. Dass ein Teil der Route über eine theoretisch schlecht befahrbare Straße führt, liegt daran, dass, wie oben beschrieben, viele Straßenabschnitte nicht vollständig attribuiert sind. Das hat zur Folge, dass gewisse Straßen nicht automatisiert eliminiert werden konnten.

\subsection{Diskussion}

Wie in den Ergebnissen erklärt, überschneiden sich einige der 3-km-Radien besonders in urbanen Gebieten, was zu Routen führen kann, die über mehr als einen Einzugskreis hinweg führen. Besonders in den Metropolregionen ist dieses Problem markant, viele Bahnhöfe liegen näher als $3 \mathrm{~km}$ zusammen, sodass sich die Radien stark überschneiden. Hier stellt sich die Herausforderung einer Routenoptimierung, die zum Beispiel mehrere mögliche Startpunkte als Teil einer Route einbezieht.

Im Kontrast dazu, sind in den ländlichen Gebieten in Bahnhofsnähe keine der ausgewählten Points of Interest, welche als Endhalt fungieren könnten, zu finden. Dies führt dazu, dass besonders in den wenig besiedelten, weitläufigen Gegenden, keine Route ermittelt werden konnte. Wenn sich ein passender Endhalt in dem 3-km-Radius befindet, 
sind die berechneten Routen im Schnitt kürzer als 4,5 km und entfallen somit im letzten Schritt des Modells, in welchem nur die Routen ausgewählt werden, die die passende Streckenlänge aufweisen. Somit fallen die besonders ländlich geprägten Regionen durch ihre Weitläufigkeit für die Befahrung durch automatisierte Shuttles weg. Dies ist allerdings auch dem Fehlen von POIs in den verfügbaren Datensätzen geschuldet, die damit nicht als mögliche Zielpunkte berücksichtigt werden konnten.

Ein anderes Problem kann die zu geringe Größe eines Dorfes darstellen, sodass sich in einem Einzugsradius mehr als ein Dorf befindet und sich somit eine Teilstrecke auf Gemeindeverbindungsstraßen befindet. Durch die derzeit nur geringe zugelassene Fahrgeschwindigkeit müssen solche Routen manuell entfernt werden, da sie zu Unfällen und Unmut in der ortsansässigen Bevölkerung führen könnten. Des Weiteren ist zu erkennen, dass Routen oftmals über den Radius der $3 \mathrm{~km}$ hinweg führen. Die Modellierung der Routen ist an die Fähigkeiten des Programms ArcGIS Pro gebunden, welches sich darauf spezifiziert, die kürzeste Route zum nächsten Zielort zu finden. Das hat als Auswirkung, dass Routen innerhalb des 3-km-Radius, im Schnitt kürzer als die vorgegebene Streckenlänge von 4,5-5,5 km sind. Somit wurden diese Strecken im letzten Schritt der Modellierung automatisch eliminiert.

\subsubsection{Risiken aus den vorhandenen Datensätzen}

Durch fehlende Attribuierungen bei offiziellen Datensätzen, sowie fehlende oder doppelte Daten in öffentlichen VGI-Datensätzen, können falsche oder unvollständige Informationen aus der Modellierung entstehen.

Die hier für das Straßennetz verwendeten Daten sind offizielle staatliche Daten. Diese werden kontinuierlich aktualisiert und auf die derzeitigen Straßenbedingungen angepasst. Allerdings sind gewisse Attributinformationen nicht vollständig vorhanden. Dies führt zu Problemen bei automatisierten Auswahlprozessen und Verarbeitungsschritten, wie sie für Modellierungen wie in diesem Kapitel beschrieben nötig sind.

Eine Möglichkeit zur Behebung solcher fehlenden Informationen ist die Aggregation verschiedener Quellen zu einer neuen Datenbasis. Nimmt man Straßendatensätze, sind diese allerdings zumeist nicht öffentlich bzw. frei zugänglich. Zusätzlich gibt es die OSM-Straßendaten, diese sind allerdings nur räumlich mit den ATKIS-Daten aggregierbar, da keine individuellen Attribute pro Straße existieren, welche die zwei Datensätze verbindbar machen würden. In diesem Fall brachte diese Aggregation der Datensätze mehr Fehler als hilfreiche Informationen hervor, weshalb von der Lösung abgesehen werden musste.

Betrachtet man die Start- und Zielorte der Routen, sind auch hier Mängel zu erkennen. OSM-Daten sind oftmals nicht auf dem neusten Stand, was dazu führen kann, dass Bahnhöfe nicht mehr in Betrieb sind, bestimmte Points of Interest nicht mehr existieren oder durch andere ersetzt wurden. Somit kann auch hier eine Fehlerquelle entstanden sein. Die Datensätze für Straßen beinhalten auch nicht alle geometrischen Informationen der 
Straßen, die sich mit den technischen Restriktionen des Fahrzeugs zu entsprechenden Parametern für die Auswahl hätten entwickeln lassen.

\subsection{2 Übertragbarkeit}

Betrachtet man die derzeitige Literatur, gibt es keine verifizierten Modelle, mit denen man Netzplanungen für den öffentlichen Personennahverkehr über einen größeren Raum automatisiert erstellen und die optimalen Routen erhalten kann. Dies liegt zum einen an der Organisation des öffentlichen Nahverkehrs in Deutschland, welcher von Gemeinde zu Gemeinde zumeist individuell geplant und teilweise auch durchgeführt wird. Zum anderen liegt es aber auch an der sehr spezifischen Struktur einer jeden Region. Informationen zum allgemeinen Verkehrsaufkommen oder der Verkehrsfrequenz von Radfahrern stehen nicht als Basisdaten zur Verfügung und können daher auch nicht in Modellen verwendet werden. Die Wahl bestimmter Strecken kann somit stark von besonderen Ortskenntnissen der Planer abhängen. Faktoren wie Frequenz und Nutzungshäufigkeit bestehender Routen, Schülerverkehre oder Sonderverkehre für Veranstaltungen konnten im Modell ebenfalls nicht berücksichtigt werden, da diese Daten nicht vorliegen. Die letzten Bedingungen kann man bei der allgemeinen Routenplanung außer Acht lassen, da es hier im ersten Schritt darum ging, Strecken zu finden, welche eine sinnvolle Route ergeben - unabhängig von der Nutzungsfrequenz. Für eine weitere Verbesserung des Modells wird sich allerdings die Frage stellen, wie Verkehrsaufkommen, die Dichte der Radfahrer oder Parkplätze am Straßenrand in ein Modell einbezogen werden können.

Die Eliminierung von Straßen mit zulässigen Höchstgeschwindigkeiten über $50 \mathrm{~km} / \mathrm{h}$ anhand des Straßentyps (Autobahn, Bundesstraße) und den anderen oben aufgeführten Attributen führt noch nicht zu einem vollständigen Ausschluss. Aufgrund der fehlenden Attribuierung einiger Straßenabschnitte, sind immer noch Strecken im Netzwerkdatensatz enthalten, welche nicht zu den vorgegebenen Parametern passen. Hier kann in zukünftigen Analysen angesetzt werden. Zusätzliche Datenquellen, alternative Erhebungsverfahren und neue Ansätze zur Verschneidung von Daten aus unterschiedlichen Datenquellen werden notwendig sein, um zu noch präziseren Aussagen zu kommen. Die derzeitige Datenlage lässt eine engere automatisierte Auswahl an Straßen auf einem so großen, heterogenen Gebiet nicht zu.

\subsubsection{Normierung im Straßenbau und Randbedingungen}

Bisherige Standards der Planung eines Verkehrssystems für den öffentlichen Personennahverkehr berücksichtigen noch nicht die Möglichkeiten und Fähigkeiten automatisierter oder autonomer Fahrzeuge. Damit bietet sich auch auf Ebene der Normierung z. B. für die bauliche Ausstattung von Verkehrsstraßen Verbesserungspotenzial. Aus Gründen der Verkehrssicherheit wird momentan eine direkte An- und Abfahrt aus Haltestellen ohne 
Rangieren oder Rückwärtsfahrt angestrebt. Aufgrund der Sensorausstattung muss bei vielen automatisierten Fahrzeugen keine Unterscheidung zwischen vorne und hinten bzw. Vor- und Rückwärtsfahrt gemacht werden. Damit wären aus baulicher Sicht auch Haltestellen in Anlehnung an Kopfbahnhöfe denkbar und somit ein Ausbau an Orten möglich, die bis dato nicht oder nur schwer bedient werden können. Auch der Aufbau von Busbahnhöfen könnte damit vereinfacht werden und der Flächenverbrauch für den Verkehr reduziert werden. Länge und Achsstände beim vorliegenden Fahrzeug sowie die Lenkung über Vorder- und Hinterachse führen zu veränderten Schleppkurven bzw. einer Verringerung der Überstreichung von Wartebereichen für Passagiere bei Ein- und Ausfahrt des Fahrzeugs in einer Busbucht. Bei einer gezielten Abstimmung von Fahrzeuggeometrie und Lenkverhalten sowie baulicher Planung von Busbuchten und Haltestellen ergäben sich auch hier Vorteile für Platzbedarf und Verkehrssicherheit.

Umgekehrt gibt es Anzeichen, dass aus Sicht der Verkehrssicherheit bzw. der gefühlten Sicherheit von Wartenden eine stärkere Kennzeichnung des Haltestellenbereichs erforderlich wird, um den Aufenthaltsraum von Personen vom Anfahrts-/Abfahrtsbereich klar abzugrenzen. Dies könnte dazu beitragen, das Risiko von Behinderungen bei $\mathrm{Zu}-$ und Abfahrt des automatisierten Fahrzeuges an Haltestellen durch Wartende im Zufahrtsbereich zu reduzieren. Gleichzeitig könnten Unsicherheiten von Wartenden bei der Anfahrt des automatisierten Fahrzeuges reduziert werden.

In Anlehnung an die in der Literatur beschriebenen Konzepte eines Car-SharingSystems mit automatisierten Pkws bietet auch das hier beschriebene Konzept eines automatisierten Shuttles neue Möglichkeiten bei der Flexibilisierung des ÖPNV-Netzes und damit einer stärker an die potenziellen bzw. fluktuierenden Bedarfe angepassten Linienführung, im Vergleich zum heute starren Haltestellen- und Taktsystem. Hierzu muss die gesetzliche Regulierung des ÖPNV, wie z. B. die Bindung an Haltestellen, sowohl rechtlich als auch organisatorisch näher untersucht und ggf. an die neuen Möglichkeiten und Bedarfe angepasst werden.

\subsection{Schlussfolgerungen und Ausblick}

Derzeitige wissenschaftliche Studien bieten einen großen Spielraum an Ideen und Vorstellungen, wie automatisierte Fahrzeuge den öffentlichen Personennahverkehr verändern und verbessern könnten. Allerdings sind diese Szenarien auf zukünftige technische und rechtliche Möglichkeiten ausgerichtet und heute noch nicht implementierbar. Weder die rechtlichen noch die technischen Bedingungen der heutigen Gesellschaft lassen Modelle wie AMOB zu. Resultierend muss die Lücke zwischen Zukunftsszenarien und dem Jetzt modelliert werden, um großflächige Einsatzpotenziale zu identifizieren und die Gesellschaft an die Nutzung automatisierter Fahrzeuge zu gewöhnen.

In diesem Kapitel wurde die Übertragbarkeit der Ergebnisse aus der Studie in Bad Birnbach überprüft. Resultate zeigen, dass im Untersuchungsgebiet Bayern viele Strecken durch die räumliche Modellierung der Parameter automatisiert in GIS identifiziert werden 
konnten. Diese Strecken stellen potenzielle neue Routen für automatisierte Shuttles unter den derzeitigen rechtlichen und technischen Randbedingungen dar. Aufgrund der vorhandenen Datenbasis konnten allerdings nicht alle zuvor identifizierten Parameter in die Modellierung mit aufgenommen werden, da bestimmte Attribute fehlen oder unvollständig sind. Die Qualität der Basisdaten stellt damit eine wichtige Grundlage für die weitere Expansion des Systems automatisierter Busse dar, solange rechtlich und technisch ein flächendeckender Einsatz eingeschränkt ist. Die Modellierung von möglichen Routen für die Anwendung automatisierter Shuttles im ÖPNV steht noch am Anfang der Forschung. Die Grundlage zur Parameterbestimmung, welche durch die langfristige Studie in Bad Birnbach gesammelt werden konnte, bietet ein großes Potenzial für weitere Modellierungsansätze. Parallel gilt es nun, neben der Erprobung der Fahrzeugkonzepte auf weiteren Strecken, neue Verfahren zu testen, um die Informationen über Straßenraum vollständiger und umfänglicher zu erfassen und damit auch die Grundlage für eine bessere Übertragbarkeit zu schaffen.

\section{Literatur}

Anu V, Walia G, Hu W, et al (2016) Effectiveness of Human Error Taxonomy during Requirements Inspection: An Empirical Investigation. pp 531-536

Braekers K, Ramaekers K, Van Nieuwenhuyse I (2016) The vehicle routing problem: State of the art classification and review. Computers \& Industrial Engineering 99:300-313. https://doi. org/10.1016/j.cie.2015.12.007

Chu KF, Lam AYS, Li VOK (2017) Dynamic lane reversal routing and scheduling for connected autonomous vehicles. In: 2017 International Smart Cities Conference (ISC2). pp 1-6

Csiszár C, Zarkeshev A (2017) Demand-capacity coordination method in autonomous public transportation. Transportation Research Procedia 27:784-790. https://doi.org/10.1016/j. trpro.2017.12.109

Cyganski R (2015) Autonome Fahrzeuge und autonomes Fahren aus Sicht der Nachfragemodellierung. In: Maurer M, Gerdes JC, Lenz B, Winner H (Hrsg) Autonomes Fahren. Springer Berlin Heidelberg, Berlin, Heidelberg, pp 241-263

Dia H, Javanshour F (2017) Autonomous Shared Mobility-On-Demand: Melbourne Pilot Simulation Study. Transportation Research Procedia 22:285-296. https://doi.org/10.1016/j. trpro.2017.03.035

Heilig M, Hilgert T, Mallig N, et al (2017) Potentials of Autonomous Vehicles in a Changing Private Transportation System - a Case Study in the Stuttgart Region. Transportation Research Procedia 26:13-21. https://doi.org/10.1016/j.trpro.2017.07.004

Koç Ç, Bektaş T, Jabali O, Laporte G (2016) Thirty years of heterogeneous vehicle routing. European Journal of Operational Research 249:1-21. https://doi.org/10.1016/j.ejor.2015.07.020

Landesamt für Digitalisierung, Breitband und Vermessung (o. J.) Geobasisdaten für Wirtschaft und Verwaltung. https://www.ldbv.bayern.de/vermessung/alkis.html, Zugriffsdatum 20. Oktober 2019.

Lenz B, Fraedrich E (2015) Neue Mobilitätskonzepte und autonomes Fahren: Potenziale der Veränderung. In: Maurer M, Gerdes JC, Lenz B, Winner H (Hrsg) Autonomes Fahren. Springer Berlin Heidelberg, Berlin, Heidelberg, pp 175-195

Meignan D, Simonin O, Koukam A (2007) Simulation and evaluation of urban bus-networks using a multiagent model. Simulation Modelling Practice and Theory 15:659-671 
Mogg T (2017) Driverless buses arrive in Paris. https://www.digitaltrends.com/cars/paris-driverless-buses/, Zugriffsdatum 20. Oktober 2019.

Neumann P (2017) Mit Tempo 20 - Autonome Busse rollen ab 2018 durch Mitte. In: Berliner Zeitung. https://www.berliner-zeitung.de/berlin/mit-tempo-20-autonome-busse-rollen-ab-2018durch-mitte-28096592, Zugriffsdatum 20. Oktober 2019.

OpenStreetMap (o. J.) OpenStreetMap - Deutschland. https://www.openstreetmap.de/, Zugriffsdatum 20. Oktober 2019.

Postauto Schweiz Medienmitteilung vom 22. Juni 2017. In: Smartshuttles feiern ihren ersten Geburtstag. https://www.postauto.ch/de/news/smartshuttles-feiern-ihren-ersten-geburtstag, Zugriffsdatum 20. Oktober 2019.

Spichkova M, Simic M, Schmidt H (2015) Formal Model for Intelligent Route Planning. Procedia Computer Science 60:1299-1308. https://doi.org/10.1016/j.procs.2015.08.196

TaBuLa (2018) Automatisiert verkehrende Busse im Kreis Herzogtum Lauenburg. In: TaBuLaAutonomes Fahren. https://vhhbus.de/tabula-autonomes-fahren/, Zugriffsdatum 20. Oktober 2019

Talebpour A, Mahmassani HS, Elfar A (2017) Investigating the Effects of Reserved Lanes for Autonomous Vehicles on Congestion and Travel Time Reliability. Transportation Research Record: Journal of the Transportation Research Board 2622:1-12. https://doi.org/10.3141/2622-01

Valdes J, Wuth J, Zink R, et al (2019) Extracting relevant Points of Interest from Open Street Map to support E-Mobility Infrastructure Models. Bavarian Journal of Applied Sciences 4:

Wiener Linien (2019) auto.Bus - Seestadt: Autonome Autobuslinie für Wien. https://www.wienerlinien.at/eportal3/ep/programView.do/pageTypeId/66528/programId/4400625/channeIId/-4400522, Zugriffsdatum 20. Oktober 2019.

wiss. Dienste Deutscher Bundestag (2018) Autonomes und automatisiertes Fahren auf der Straße rechtlicher Rahmen 
Open Access Dieses Kapitel wird unter der Creative Commons Namensnennung 4.0 International Lizenz (http://creativecommons.org/licenses/by/4.0/deed.de) veröffentlicht, welche die Nutzung, Vervielfältigung, Bearbeitung, Verbreitung und Wiedergabe in jeglichem Medium und Format erlaubt, sofern Sie den/die ursprünglichen Autor(en) und die Quelle ordnungsgemäß nennen, einen Link zur Creative Commons Lizenz beifügen und angeben, ob Änderungen vorgenommen wurden.

Die in diesem Kapitel enthaltenen Bilder und sonstiges Drittmaterial unterliegen ebenfalls der genannten Creative Commons Lizenz, sofern sich aus der Abbildungslegende nichts anderes ergibt. Sofern das betreffende Material nicht unter der genannten Creative Commons Lizenz steht und die betreffende Handlung nicht nach gesetzlichen Vorschriften erlaubt ist, ist für die oben aufgeführten Weiterverwendungen des Materials die Einwilligung des jeweiligen Rechteinhabers einzuholen.

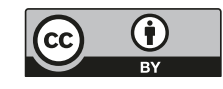

